\title{
Padrones de Distribución del Ramo Superficial del Nervio Radial en el Dorso de la Mano Humana
}

\author{
Distribution Patterns of the Superficial Branch of the \\ Radial Nerve in the Back of the Human Hand
}

Tiznado, G.*; Olave, E.** \& Sousa-Rodrigues, C.****

TIZNADO, G.; OLAVE, E. \& SOUSA-RODRIGUES, C. Padrones de distribución del ramo superficial del nervio radial en el dorso de la mano humana. Int. J. Morphol., 31(3):857-863, 2013.

RESUMEN: La inervación sensitiva del dorso de la mano está dada generalmente por el ramo superficial del nervio radial (RSNR) y el ramo dorsal del nervio ulnar (RDNU). Con el propósito de obtener una descripción detallada del origen, trayecto, distribución y ramificación del RSNR, como también elaborar una padronización del surgimiento de los nervios digitales dorsales de la mano, se estudiaron 36 manos de cadáveres formolizados, 30 de individuos adultos brasileños y 6 de chilenos. El RSNR se hizo superficial a una distancia de $89,4 \pm 12,1 \mathrm{~mm}$ proximal al nivel del proceso estiloides radial (PER) y se dividió en un ramo ventral y un ramo dorsal a 57,6 $\pm 17,8$ mm proximal al PER. Posteriormente el ramo dorsal del RSNR se dividió en un ramo radial y un ramo ulnar a 4,9 $\pm 14,7$ mm proximal al PER. Se realizó una clasificación en 4 padrones, según el origen de los nervios digitales dorsales proporcionados por los ramos principales del RSNR. El padrón I se observó en 75\% de los casos, en tanto que el padrón II se halló en el 13,89\%, el padrón III y IV se observaron en el 5,56\% de las muestras, respectivamente. Se evidenció una comunicación entre el RSNR y el RNDU en el 58,3\% de las muestras. El conocimiento de la disposición de los ramos sensitivos dorsales de la mano es de importancia para la anatomía quirúrgica de la región dorsal de la muñeca y la mano, ya que es un sector donde se realizan procedimientos que podrían poner en riesgo a los nervios mencionados.

PALABRAS CLAVE: Anatomía; Mano; Nervio radial; Ramo superficial.

\section{INTRODUCCIÓN}

La obtención de conocimientos detallados sobre la anatomía del dorso de la mano, ha sido una tarea que han desarrollado diversos autores durante las últimas décadas, especialmente con el propósito de evitar daños que pudiesen conducir a lesiones como parestesias, hiperestesias o neuromas dolorosos durante el abordaje de procedimientos quirúrgicos (Hazari \& Elliot, 2004).

La inervación sensitiva del dorso de la mano está asociada principalmente a ramos provenientes del ramo superficial del nervio radial y del ramo dorsal del nervio ulnar, donde según Williams et al. (1995) la distribución del primero, abarcaría la zona desde el pulgar a la mitad radial del dedo anular. Sin embargo, Rouvière \& Delmas (2002) indican que la distribución del primero comprendería la zona desde el pulgar a la mitad radial del dedo medio.

Como los tratados clásicos y ulteriores estudios anatómicos no han mostrado una descripción detallada de la disposición y topografía de los nervios en el dorso de la mano, el propósito de esta investigación ha sido estudiar el origen, trayecto, distribución y ramificación del ramo superficial del nervio radial, con el fin de obtener una padronización y las variaciones del mismo, contribuyendo de esta manera al conocimiento morfológico, que permitan ayudar tanto a la clínica, como a las técnicas quirúrgicas actuales.

\section{MATERIAL Y MÉTODO}

El estudio fue realizado en 30 miembros superiores de individuos adultos Brasileños y 6 miembros superiores de individuos adultos Chilenos, fijados en solución acuosa de formaldehido al 10\%, de los cuales 16 eran miembros del lado derecho y 20 del lado izquierdo. Los primeros estaban localizados en los Laboratorios de Anatomía de la Universidade Estadual de Ciencias da Saúde de Alagoas,

\footnotetext{
* Universidad San Sebastián, Sede Osorno, Chile.

** Universidad de La Frontera, Temuco, Chile.

***Universidade Estadual de Ciencias da Saude; Universidade Federal de Alagoas, Maceió, Brasil.
} 
Maceió, Brasil y los segundos en los Laboratorios de Anatomía de la Facultad de Medicina de la Universidad de la Frontera, Temuco, Chile.

Se efectuó la disección del antebrazo y dorso de cada una de las manos, con ayuda de una lupa Ranson con aumento de 3x para facilitar la identificación de estructuras finas. Se retiró la piel y el tejido adiposo subcutáneo, evidenciando el ramo superficial del nervio radial (RSNR) y sus ramos, clasificando a éstos para su padronización. Todas las muestras fueron fotografiadas y esquematizadas con una cámara digital Panasonic Lumix ZS8 de 14,1 mega pixeles marca Panasonic.

\section{RESULTADOS}

En todas las muestras observamos que el RSNR descendió por el tercio medio del antebrazo, lateralmente a la arteria radial y cubierto por el músculo braquiorradial, hasta llegar al tercio distal del antebrazo para emerger a la fascia superficial entre los tendones del músculo braquiorradial y el extensor radial del carpo. El RSNR se hizo superficial a una distancia de $89,4 \pm 12,1 \mathrm{~mm}$ proximal al proceso estiloides radial (PER) y prosiguió en dirección al dorso de la mano, dividiéndose proximal al proceso estiloides del radio en un ramo ventral y un ramo dorsal, a $57,6 \pm 17,8 \mathrm{~mm}$. El primero siguió un trayecto lateral y palmar a este proceso, continuando por la cara radial del carpo y del primer hueso metacarpiano. En tanto, que el ramo dorsal continuó hacia el dorso de la mano, dividiéndose a $4,9 \pm 14,7 \mathrm{~mm}$ proximal al PER, en un ramo radial $\mathrm{y}$ otro ulnar.

En 18 casos (50\%) esta división ocurrió proximal al PER, distal en 15 casos $(41,7 \%)$ y en 3 casos al mismo nivel. En 34 de los 36 casos estudiados $(94,4 \%)$, los ramos del RSNR se distribuyeron en la porción lateral del dorso de la mano (entre el primer y segundo espacio interóseo) y las falanges proximales del primer, segundo y tercer dígito, y en los 2 casos restantes (5,6\%), se distribuyeron por todo el dorso de la mano y por todas las falanges proximales de los cinco dígitos.

Basados en las observaciones de la distribución de este nervio en el dorso de la mano, clasificamos su ramificación en distintos grupos, según el origen de los nervios digitales dorsales.

Según el criterio anteriormente expuesto, la distribución del ramo superficial del nervio radial se clasificó en 4 padrones:
Padrón I. Esta disposición se observó en 27 miembros (75\%, Figs. 1 y 2), de los cuales 13 eran de manos derechas y 14 de manos izquierdas. En este padrón, el ramo ventral siguió un recorrido palmar al PER continuando por el margen lateral del carpo y del primer metacarpiano llegando hasta la falange proximal del pulgar, donde conformó el nervio digital dorsal radial del pulgar (R1). El ramo dorsal, en tanto, continuó distalmente, pasando en todos los casos, entre el tubérculo dorsal del radio (TDR) y el PER hasta bifurcarse en 11 casos proximal al PER, en 13 casos distal y en 3 casos al mismo nivel, en ramos radial y ulnar. El ramo radial siguió un recorrido por el dorso de la mano hacia el primer espacio interóseo, bifurcándose en promedio a 26,3 mm (DE:11,3 ) distal al PER; en un ramo medial que continuó hacia la cara dorsoulnar del pulgar, para conformar el nervio digital dorsal ulnar del pulgar (C1) y otro ramo lateral que se dirigió a la cara dorsorradial del dedo indicador, para conformar el nervio digital dorsal radial del indicador (R2). Por su parte, el ramo ulnar constituyó un tronco común (TC) que prosiguió en dirección oblicua hacia el segundo espacio interóseo dividiéndose en promedio a 67,2 mm (DE:13,7) distal al PER, en un ramo medial que conformaría el nervio digital dorsal ulnar del indicador (C2) y un ramo lateral que conformaría el nervio digital dorsal radial del dedo medio (R3).

Dentro de este grupo, en 14 muestras, el ramo ulnar del ramo dorsal del RSNR emitió un ramo comunicante (RC), que se originó en promedio a 22,2 $\mathrm{mm}$ (DE 17,1 mm) distal al PER. Éste continuó en sentido distal y oblicuo hacia el tercer espacio interóseo, donde se unió con el ramo lateral del RDNU, en un punto distal al proceso estiloides de la ulna, que estuvo a un promedio de 63,9 $\mathrm{mm}$ (DE 18,1).

Padrón II. Esta disposición se observó en 5 miembros $(13,89 \%$, Figs. 1 y 3$)$ de los cuales 4 eran de manos izquierdas y sólo una de mano derecha. En este padrón el ramo ventral siguió un recorrido lateral y palmar al PER, continuando por la parte lateral del carpo y del primer metacarpiano, para llegar a la primera falange proximal, donde conformó el nervio digital dorsal radial del pulgar (R1). En tanto el ramo dorsal, en todos los casos se dividió proximal al PER dando origen al ramo radial y al ramo ulnar. El ramo radial, se dirigió distalmente, pasando dorsal y medial al PER, por el margen medial del primer metacarpiano, llegando a la primera falange distal sin dividirse, donde conformó el nervio digital dorsal ulnar del pulgar (C1). El ramo ulnar, por su parte, casi inmediatamente después de su origen, se dividió, en un ramo lateral, el cual distalmente conformaría el nervio digital dorsal radial del dedo indicador (R2) y un ramo medial. Este último, a una distancia promedio de 30,4 mm (DE: 15,1) distal al PER proporcionó un ramo comunicante, que se dirigió distal y oblicuo hacia el tercer espacio interóseo, donde se comuni- 
có con el ramo lateral del ramo dorsal del nervio ulnar (RDNU), en un punto que se encontró a una distancia promedio de 75,2 mm (DE: 6,5) distal a la línea del PEU. Posterior al origen de $\mathrm{RC}$, el ramo medial continuó como un tronco común (TC) hacia el segundo espacio interóseo, donde se dividió en el nervio digital dorsal ulnar del dedo indicador (C2) y el nervio digital dorsal digital radial del dedo anular (R3). Esto tuvo lugar a una distancia promedio de 82,4 mm (DE: 15,5) distal al PER.

Padrón III. Esta disposición se observó en los dos miembros (5,56\%, Fig. 1) de un mismo cadáver. En este padrón, el ramo ventral se dividió a una distancia promedio de $23 \mathrm{~mm}$ (DE: 2,8 ) proximal al PER, en un ramo lateral, que transcurrió ventral al PER y siguió por el margen radial del carpo y del primer metacarpiano hasta conformar el nervio digital dorsal radial del pulgar (R1), y un ramo medial, que discurrió dorsal al PER y continuó hasta el margen medial del primer metacarpiano, para distalmente conformar el nervio digital dorsal ulnar del pulgar (C1). Por otro lado, el ramo dorsal alcanzó el dorso de la mano, pasando en los dos casos, entre el TDR y el PER, hasta bifurcarse, en ambos casos, distal al PER en un ramo radial y un ramo ulnar. El primero discurrió por el primer espacio interóseo, lateral al margen del segundo metacarpiano, para distalmente conformar el nervio digital radial del indicador (R2). En tanto, el ramo ulnar proporcionó un RC, que se originó a una distancia promedio de 19,5 mm (DE: 12) distal al PER, que se comunicó con el ramo lateral del RDNU, a una distancia promedio de $54,5 \mathrm{~mm}$ (DE: 9,2) distal al PEU. Posteriormente continuó como un tronco común (TC) hacia el segundo espacio interóseo, lugar donde se dividió en el nervio digital dorsal ulnar del dedo indicador (C2) y el nervio digital dorsal radial del dedo medio (R3). Esta división ocurrió a una distancia promedio de $78 \mathrm{~mm}$ (DE: 4,2) distal al PER.

Padrón IV. Esta disposición se observó en dos miembros (5,56\%, Fig. 1), de cadáveres, nacionalidades y de lados distintos. En esta disposición se observó que la inervación sensitiva del dorso de la mano fue proporcionada exclusivamente por el RSNR, sin presencia de ramos del nervio ulnar.

En este padrón el ramo ventral transcurrió lateral y ventral al PER y continuó por el margen lateral del primer metacarpiano, llegando a la primera falange proximal, para conformar el nervio digital dorsal radial del pulgar (R1). El ramo dorsal continuó hacia el dorso de la mano donde se bifurcó en un ramo radial y en un ramo ulnar, ambos proximales al PER. El ramo radial prosiguió su recorrido, pasando entre el PER y el TDR, hacia el primer espacio interóseo, donde se bifurcó en el nervio digital dorsal ulnar del pulgar (C1) y el nervio dorsal radial del dedo indicador (R2). Esta división se produjo en promedio a 36,5 mm (DE:7) distal al PER.

Por su parte, el ramo ulnar dio origen a tres ramos (lateral, intermedio y medial), donde el más lateral continuó hacia el segundo espacio interóseo, dividiéndose a una distancia promedio de $50 \mathrm{~mm}$ (DE: 12,7) distal al nivel del PER, en el nervio dorsal ulnar del dedo indicador (C2) y el nervio digital dorsal radial del dedo medio (R3). El ramo intermedio se dirigió hacia el tercer espacio interóseo, dividiéndose en promedio, a una distancia de $57 \mathrm{~mm}$ (DE: 1,4) distal al
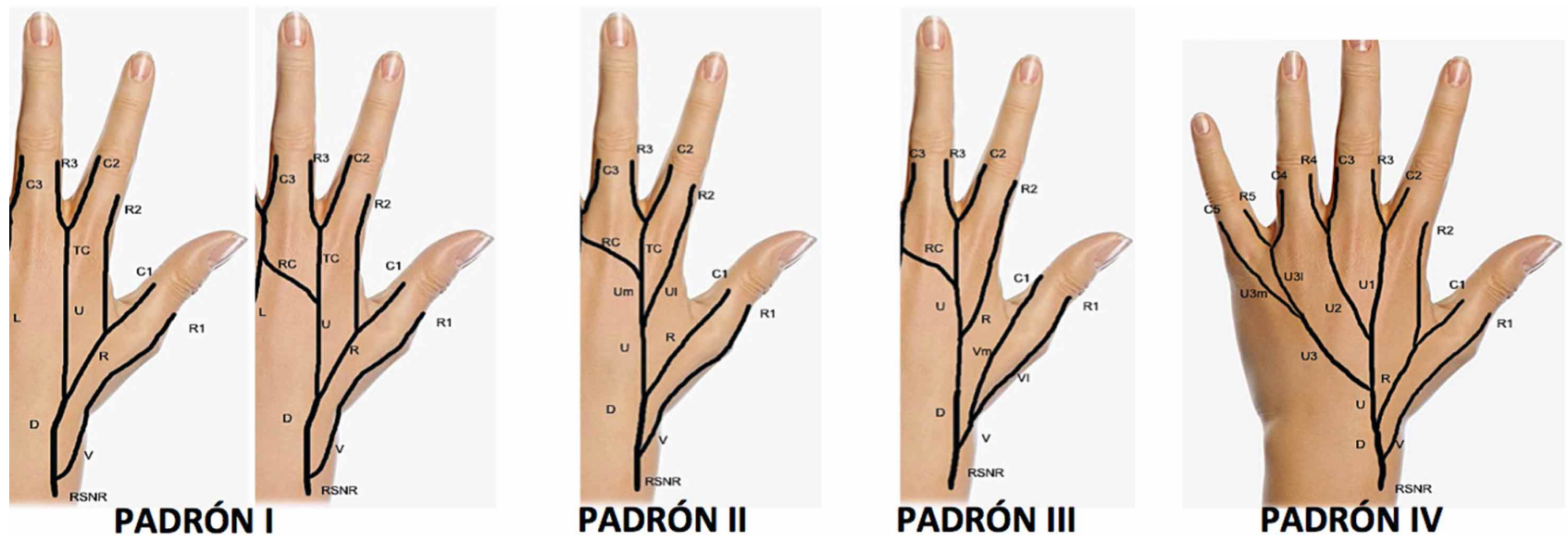

Fig. 1. Esquema sobre los padrones de distribución del ramo superficial del nervio radial en el dorso de la mano humana. RSNR, ramo superficial del nervio radial; V, ramo ventral; D, ramo dorsal; $\mathrm{R}$, ramo radial; $\mathrm{U}$, ramo ulnar; RC, ramo comunicante; TC, tronco común; Um, ramo medial del ramo ulnar; U1, ramo lateral del ramo ulnar; R1, nervio digital dorsal radial del dedo pulgar; C1, nervio digital dorsal ulnar del dedo pulgar; R2, nervio digital dorsal radial del dedo indicador; C2, nervio digital dorsal ulnar del dedo indicador; R3: nervio digital dorsal radial del dedo medio; C3, nervio digital dorsal ulnar del dedo medio; R4, nervio digital dorsal radial del dedo anular; C4, nervio digital dorsal ulnar del dedo anular; R5, nervio digital dorsal radial del dedo mínimo; C5, nervio digital dorsal ulnar del dedo mínimo. En el padrón IV, U1,U2,U3, ramos ulnares; U3m y U31, ramos medial y lateral. 
TIZNADO, G.; OLAVE, E. \& SOUSA-RODRIGUES, C. Padrones de distribución del ramo superficial del nervio radial en el dorso de la mano humana. Int. J. Morphol., 31(3):857-863, 2013.

PER, en el nervio digital dorsal ulnar del dedo medio (C3) y el nervio digital dorsal radial del dedo anular (R4). En tanto que el ramo medial, continuó un recorrido más oblicuo hacia el cuarto espacio interóseo, donde se bifurcó en un ramo lateral y un ramo medial, a una distancia promedio de 18,5 mm (DE:10,6) distal al PER. El primero de éstos, continuó distalmente dividiéndose en el nervio digital dorsal ulnar del dedo anular (C4) y el nervio digital dorsal radial del

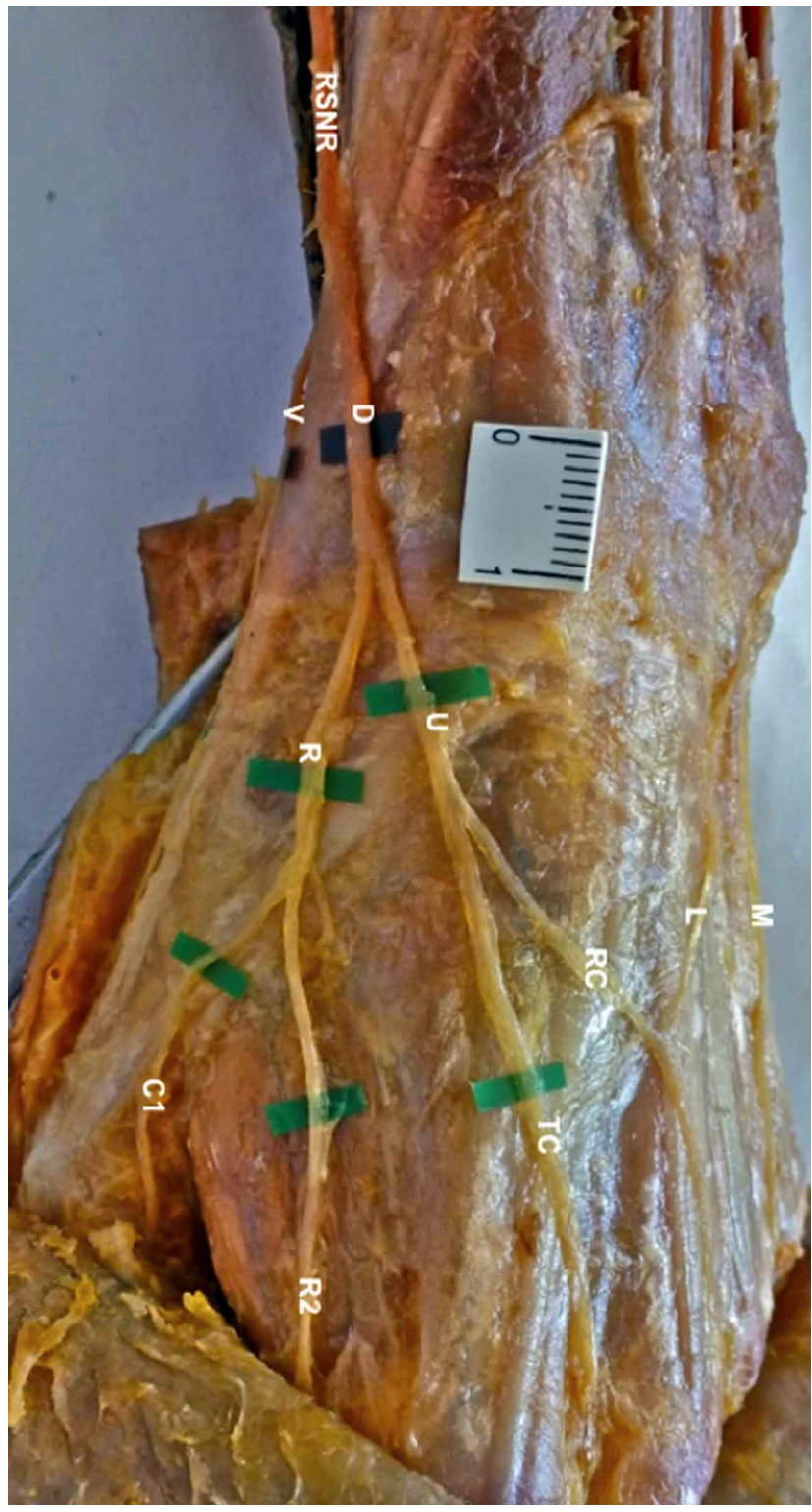

Fig. 2. Aspecto dorsorradial de una mano izquierda. Ramo superficial del nervio radial (RSNR); ramo ventral (V); ramo dorsal (D); ramo radial $(\mathrm{R})$; ramo ulnar $(\mathrm{U})$; ramo comunicante $(\mathrm{RC})$; tronco común (TC); ramo lateral del ramo dorsal del nervio ulnar (L); ramo medial del ramo dorsal del nervio ulnar (M); nervio digital dorsal ulnar del pulgar (C1); nervio digital dorsal radial del dedo indicador (R2). dedo meñique (R5), mientras que el segundo continuó hacia la parte lateral del quinto dedo para conformar el nervio digital dorsal ulnar del dedo mínimo (C5).

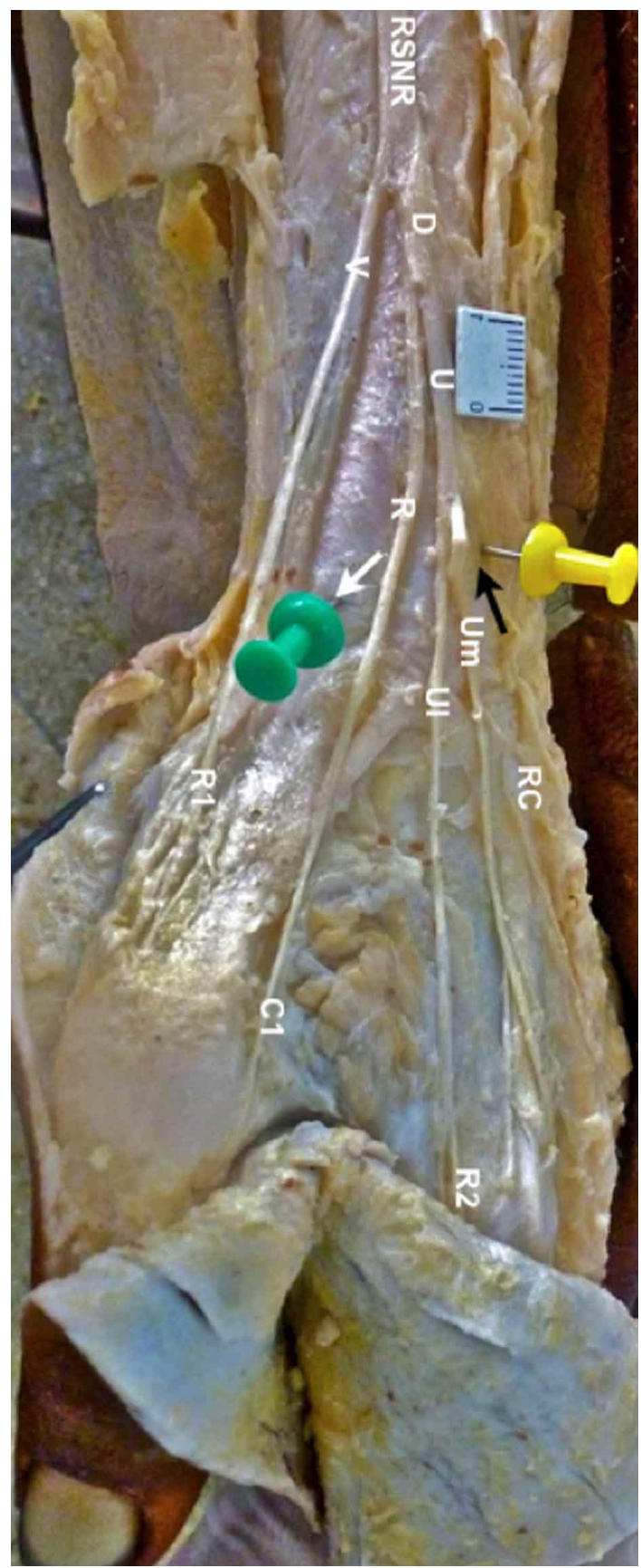

Fig. 3. Zona radial del dorso de una mano izquierda. Ramo superficial del nervio radial (RSNR); ramo ventral (V); ramo dorsal (D); ramo radial (R); ramo ulnar (U); ramo lateral (Rl); ramo medial (Rm); ramo comunicantes (RC); nervio digital dorsal radial del pulgar (R1); nervio digital ulnar del pulgar $(\mathrm{C} 1)$; nervio digital radial del dedo indicador (R2). La flecha negra indica la ubicación del tubérculo dorsal del radio; la flecha blanca indica la ubicación del proceso estiloides del radio. 
Considerando la disposición general de los nervios digitales dorsales emitidos por el RSNR se observó que el ramo ventral del RSNR proporcionó el nervio digital dorsal radial del dedo pulgar en $94,4 \%$ de los casos y a éste más el nervio digital dorsal ulnar del mismo dedo en 5,6\%. Por su parte, el ramo dorsal del RSNR, a través de su ramo radial, dio origen al nervio digital dorsal ulnar del dedo pulgar y al nervio digital dorsal radial del dedo indicador en $80,6 \%$ de las muestras, mientras que su ramo ulnar emitió el nervio digital dorsal ulnar del dedo indicador y al nervio digital dorsal radial del dedo medio en $94,4 \%$. En dos casos especiales $(5,6 \%)$, el ramo dorsal emitió todos los nervios digitales dorsales excepto el ramo digital dorsal radial del dedo pulgar que fue otorgado por el ramo ventral.

\section{DISCUSIÓN}

Actualmente los tratados clásicos carecen de una descripción detallada de las estructuras comprometidas en la sensibilidad del dorso de la mano, siendo a veces descripciones contradictorias. Muchos procedimientos quirúrgicos que son realizados en esta parte del cuerpo, necesitan de la ejecución de una incisión o aplicación de fijación externa a través de agujas (Ropars et al., 2010), que podrían lesionar estructuras nerviosas que forman parte del ramo superficial del nervio radial o el ramo dorsal del nervio ulnar.

Por esta razón, el conocimiento del origen, del punto en que se hace superficial y la ramificación de estos nervios, deben ser minuciosamente descritos. Esto ayudaría a la prevención de lesiones ocasionados en cirugías abiertas y artroscópicas (McAdams \& Hentz, 2002), que pudiesen llevar a la formación de neuromas dolorosos o compromisos en la sensibilidad de la zona (Hazari \& Elliot).

La clásica descripción sobre la inervación sensitiva del dorso de la mano, es atribuida a ramos procedentes del nervio radial y ulnar, en que el primero se encarga de la inervación lateral del dorso de la mano y el segundo del aspecto medial del dorso de la mano, incluyendo las falanges proximales de los dedos. Al revisar los tratados clásicos de anatomía, se puede apreciar como diferentes autores tienen conceptos distintos del área que abarca cada uno de estos nervios. Por ejemplo Testut \& Latarjet (1972); Chatain \& Bustamante (1986); Sinelnikov (1986); Williams et al.; Moore \& Dalley (2002); Latarjet \& Ruiz Liard (2004); Llusá et al. (2204) y Rouvière \& Delmas han señalado que el RSNR y el RDNU proporcionan el 50\% cada uno de los nervios digitales dorsales. Sin embargo Ellis (2006) describió que el RSNR proporciona los nervios digitales dorsales del 1er, $2 \mathrm{do}$, 3er y el aspecto radial del 4to dedo, en tanto que el
RDNU proporcionaría los nervios digitales dorsales del $5^{\circ}$ y parte ulnar del $4^{\circ}$ dedo. Por otro lado Leversedge et al. (2010) son más específicos al detallar que el $45 \%$ de los casos, el RSNR inerva $21 / 2$ de los dedos radiales y en el $30 \%$ de los casos inerva los $31 / 2$ dedos radiales.

Se demostró en nuestro estudio que en la mayoría de los casos el RSNR proporcionó los nervios digitales dorsales del 1er, 2do y aspecto radial del 3er dedo $(94,4 \%)$, lo que es consistente con lo publicado por Tellioglu et al. (2000), quienes encontraron esta misma disposición en todas las muestras estudiadas. Así mismo como Vergara-Amador \& Nieto (2010), encontraron esta disposición en el 56\% de los casos, señalando también que en el 28\% de las muestras el RSNR proporcionó los nervios digitales a los 3 dedos radiales, en el 12\% a 31/2 dedos radiales y en el $4 \%$ a $41 / 2$ dedos radiales. No obstante, estas disposiciones no fueron observadas en ninguna muestra de nuestro estudio. Por su parte Zaki (2006) indicó que en el 40\% de las muestras, el RSNR proporcionó los nervios digitales dorsales a los 3 dedos radiales, solo en 35\% de los casos el RSNR proporcionó a los $21 / 2$ dedos radiales y en el $25 \%$ restante a los $31 / 2$ dedos radiales, en tanto que Robson et al. (2008) reportaron que en todas las muestras analizadas el RSNR entregó ramos a los 3 dedos radiales.

Es importante señalar que en nuestra serie se encontró una rara variación de la inervación del dorso de la mano, casos ya publicados por Tiznado et al. (2012), disposición que también fue mencionada por Tountas \& Bergman (1993). En dos muestras $(5,4 \%)$ observamos que el nervio radial "invadió" el territorio que generalmente es inervado por el RDNU, proporcionando la inervación total del dorso de la mano, además de proporcionar todos los nervios digitales dorsales. En este caso no hubo presencia del RNDU en el dorso de la mano, contrario a lo reportado por los autores antes mencionados, quienes hallaron que los nervios digitales dorsales, que no fueron proporcionados por RSNR, fueron suministrados en su totalidad por el RDNU.

Diversos estudios, al igual que el nuestro, describen detalladamente el origen de los nervios digitales dorsales proporcionados por los diferentes ramos del RSNR. En la totalidad de los casos descritos por Tellioglu et al. y Bianchi (2002), y en la mayoría de los casos (65\%) según Zaki; Vergara-Amador \& Nieto (92\%); Chen et al. (2010) (95\%), e incluyendo nuestro estudio (94,4\%), el ramo ventral del RSNR proporcionó únicamente el nervio digital dorsal radial del dedo pulgar, demostrando que esta situación sería la normalidad. En tanto, que en una proporción mucho menor, el ramo ventral del RSNR suministró tanto el nervio digital dorsal radial como el nervio digital dorsal ulnar del pulgar, revelando que esta situación correspondería a una variación 
anatómica (Zaki, 35\%; Vergara-Amador \& Nieto, 8\%; nuestro estudio, $5,6 \%$ ).

No obstante, al observar los nervios digitales dorsales proporcionados por el ramo dorsal del RSNR, se puede concluir que hay una mayor diversidad en las disposiciones descritas por los autores consultados, lo que no ayuda a evidenciar cual sería la normalidad anatómica. Como Tellioglu et al. quienes especificaron que en la totalidad de los casos, y en la gran mayoría de los casos (80,6\%), según nuestro estudio, el ramo radial del ramo dorsal del RSNR suministró tanto, el nervio digital dorsal ulnar del pulgar como el nervio digital dorsal radial del dedo indicador. Sin embargo, esto no es compartido por Bianchi, quien encontró que esta disposición no alcanzaba el 50\% de los casos, mientras tanto que Zaki detalló esta situación, en una proporción aún menor (30\%).

En cuanto a los nervios digitales dorsales proporcionados por el ramo ulnar del ramo dorsal del RSNR, también se percibe gran diversidad de padrones. Tellioglu et al. reportaron que en la totalidad de los casos, el ramo ulnar del ramo dorsal del RSNR proporcionó el nervio digital dorsal ulnar del dedo indicador, asimismo como el nervio digital dorsal radial del dedo medio. Esta disposición también fue observada en la gran mayoría de los casos analizados en nuestro estudio $(94,4 \%)$. Sin embargo, esto no es compartido por Bianchi y Zaki, quienes encontraron una prevalencia bastante menor al 50\%, de esta disposición (11\% y 25\%, respectivamente).

Otras disposiciones también fueron descritas por estos últimos autores, donde este ramo dio origen a los nervios digitales dorsales radial y ulnar del dedo indicador y al nervio digital dorsal radial del dedo medio (Bianchi, 19\% y Zaki, 40\%). La falta de un padrón común hace pensar que se puede deber a la escasa cantidad de muestra indicadas en los estudios o a la gran variación que existiría entre diferentes individuos.

Los resultados expuestos en la presente investigación sirven para configurar una padronización más uniforme de la inervación sensitiva del dorso de la mano, llevada a cabo principalmente por el ramo superficial del nervio radial y el ramo dorsal del nervio ulnar.

Teniendo en cuenta, que siempre en anatomía y especialmente en cirugía, se debe estar atento a las eventuales variaciones anatómicas, una descripción más detallada de las estructuras descritas, proporcionaría mayor protección sobre los diversos ramos que se distribuyen en esta zona del cuerpo.

TIZNADO, G.; OLAVE, E. \& SOUSA-RODRIGUES, C. Distribution patterns of the superficial branch of the radial nerve in the back of the human hand. Int. J. Morphol., 31(3):857-863, 2013.

SUMMARY: The sensory innervation of the dorsum of the hand is usually given by the superficial branch of radial nerve (SBRN) and the dorsal branch of the ulnar nerve (DBUN). In order to obtaining a detailed description of the origin, course, distribution and the branching of the SBRN, and also to develop a patterning of the emergence of dorsal digital nerves of the hand, we made a dissection study on dissection of cadaver specimens, 36 hands, 30 adult Brazilians and 6 Chileans. The SBRN became superficial at a distance of $89,4 \pm 12,1 \mathrm{~mm}$ proximal to the level of styloid process of the radius (SPR) and it branched in a ventral and dorsal branch at a distance of $57,6 \pm 17,8 \mathrm{~mm}$ proximal to the level of SPR. Later the dorsal branch of SBRN branched in a radial and ulnar branch at a distance of 4,9 $\pm 14,7 \mathrm{~mm}$ proximal to the level of SPR. It was performed a classification of four pattern, according to the source of the dorsal digital nerves provided by the main branches of SBRN. The pattern I was present in $75 \%$ of the cases, while the pattern II was found in $13,89 \%$, patterns III and IV were observed in 5,56\% of the samples, respectively. It was observed a communication between SBRN and DBUN in 58,3\% of the samples. Knowledge of the arrangement of the sensitive dorsal branches is of great importance to the surgical anatomy of the dorsal region of the wrist and hand, where procedures are conducted, that could jeopardize the nerve and its branches.

KEY WORDS: Anatomy; Hand; Radial nerve; Superficial branch

\section{REFERENCIAS BIBLIOGRÁFICAS}

Bianchi, H. Ramification of the superficial branch of the radial nervus. Rev. Chil. Anat., 20(3):247-50, 2002.

Chatain, I. \& Bustamante, J. Anatomía macroscópica, funcional y clínica. México D. F., Sistemas Técnicos de Edición, 1986, p.820.
Chen, Y.; Zheng, X.; Wang, J.; Zhu, Y. \& Zhan, C. Reliable techniques to avoid damaging the superficial radial nerve due to percutaneous Kirschner wire fixation of the distal radius fracture through the radial styloid process. Surg. Radiol. Anat., 32(8):711-7, 2010. 
Ellis, H. Clinical Anatomy: applied anatomy for students and junior doctors. 11th ed. Massachusetts, Blackwell Publishing, 2006,439 p.

Hazari, A. \& Elliot, D. Treatment of end neuromas, neuromas in continuity and scarred nerves of the digits by proximal relocation. J. Hand Surg. Br., 29(4):338-50, 2004.

Latarjet, M. \& Ruiz Liard, A. Anatomía humana. $4^{\mathrm{a}}$ Ed. Buenos Aires, Médica Panamericana, 2004, p.896.

Leversedge, F.; Goldfarb, C. \& Boyer, M. A pocketbook Manual of hand and upper extremity anatomy: Primus manus. Philadelphia, Lippincott Williams \& Wilkins, 2010. p.136p.

Llusá, M.; Merí, A. \& Ruano, D. Manual y atlas fotográfico de anatomía del aparato locomotor. Buenos Aires, Panamericana, 2004.

McAdams, T. R. \& Hentz, V. R. Injury to the dorsal sensory branch of the ulnar nerve in the arthroscopic repair of ulnar-sided triangular fibrocartilage tears using anin side-out technique: A cadaver study. J. Hand Surg. Am., 27(5):840-4, 2002.

Moore, K. \& Dalley, A. Anatomía con orientación clínica. 4a Ed. Buenos Aires, Médica Panamericana, 2002. p.1185.

Robson, A.; See, M. S. \& Ellis, H. Applied anatomy of the superficial of the radial nerve. Clin. Anat., 21(1):38-45, 2008.

Ropars, M.; Fontaine, I.; Morandi, X.; Berton, E.; Kaila, R. \& Darnault, P. Preserving the superficial branch of the radial nerve during carpometacarpal and metacarpophalangeal joint arthroscopy: an anatomical study. Surg. Radiol. Anat., 32(3):271-6, 2010.

Rouvière, H. \& Delmas, A. Anatomía humana: descriptiva, topográfica y funcional. 10 Ed. Barcelona, Masson, 2002, p.755.

Sinelnikov, R. Atlas de anatomía humana. $4^{\mathrm{a}}$ ed. Moscú, MIR, 1986. p.397.

Tellioglu, A. T.; Tekdemir, I.; Ersoy, M. \& Karabag, O. Dorsal branches of superficial radial nerve: an anatomic study with potential clinical applications. Eur. J. Plast. Surg., 23(8):41921, 2000.

Testut, L. \& Latarjet, A. Tratado de anatomía humana. $9^{\mathrm{a}}$ ed. Barcelona, Salvat, 1972. p.1142.

Tiznado, G.; Sousa-Rodigues, C. \& Olave, E. Ramo superficial del nervio radial: amplia distribución en el dorso de la mano. Int. J. Morphol., 30(2):374-8, 2012.

Tountas, C. \& Bergman, R. Anatomic variations of the upper extremity. New York, Churchill \& Livingstone Inc., 1993. p. 286 .
Vergara-Amador, E. \& Nieto, J. L. Estudio anatómico de la rama superficial del nervio radial. Implicaciones quirúrgicas. Rev. Fac. Med., 58(3):214-20, 2010.

Williams, P. L.; Warwick, R.; Dyson, M. \& Bannister, L. H. Gray Anatomía. 37ª ed. Rio de Janeiro, Guanabara-Koogan, 1995.

Zaki, A. Anatomical study of the superficial branch of the radial nerve. Bull. Alex. Fac. Med., 42(2):525-30, 2006.

Dirección para Correspondencia:

Prof. Dr. Enrique Olave

Facultad de Medicina

Universidad de La Frontera

Casilla $54 \mathrm{D}$

Temuco - CHILE

Email: enrique.olave@ufrontera.cl

Recibido : 18-01-2013

Aceptado: 22-05-2013 